Military Technical College, Kobry El-Kobbah, Cairo, Egypt

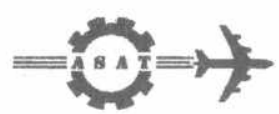

$9^{\text {th }}$ International Conference On Aerospace Sciences \& Aviation Technology

\title{
The Effect of Satellite Diversity on Service Availability, Blockage Probability and System Capacity over Rician-Lognormal Channel of NGEO Satellite Systems
}

\author{
Tarek Attia*, Peter Sweeney ${ }^{\star \star}$ and Martin Sweeting ${ }^{\star \star *}$
}

\begin{abstract}
In this paper, we analyse the performance of the downlink of a Non-Geostationary Earth Orbits (NGEO) mobile satellite channel based on Direct Sequence-Code Division Multiple Access (DS-CDMA) in the presence of shadowing, fading and interference from other satellites visible at the same time. In particular, the performance of QPSK convolutionally coded DS-CDMA over a Rice-Lognormal channel is analysed in depth. The provision of such a service requires that the user must have sufficient link quality for the duration of call. To have sufficient link quality, the user must have an adequate power to overcome the path loss and other physical impairments and maintain the performance of the system. The most important factor in determining the performance is the probability of signal shadowing which depends both on the user environment and on the constellation.
\end{abstract}

The paper addresses the exploitation of satellite diversity in the Rician-Lognormal channel. In particular, we focus on the impact of satellite diversity on service availability, path blockage, call dropping and system capacity, considering the effects of imperfect power control, shadowing and voice activity. Concatenated codes are used to provide different bit error probabilities for different services.

We show that satellite diversity is an essential in providing service availability especially in urban and suburban areas. Also we show the impact of satellite diversity on the system capacity under different environments, which is related to elevation angle, latitude and the nature of the user terminal environment.

\section{KEYWORDS:}

Satellite communication, Non-Geostationary Earth orbit and DS-CDMA.

* PhD. Student at Surrey Space Centre, University of Surrey

** Senior lecturer at Centre for Communication Systems Research

*** Professor and Director of Surrey Space Centre 


\section{Introduction}

The trend for satellite system architectures aimed at providing personal mobile services is the deployment of multi-satellite constellations in NGEO. This allows the user terminals to be small size, low cost and low power demanding.

In present and next generation satellite systems, CDMA has been proposed as the multiple access technique for a number of mobile satellite communication systems. Some of the proposed systems adopt CDMA to take advantage of its properties of interference resilience, robustness against shadowing and fading, spotbeam and satellite soft handover, path diversity exploitation, possibility of spectrum sharing, simplicity in resource management, low probability of intercept [1-2]. The new MSS will provide communications to both developed and developing areas of the world where there is little or no telecommunications infrastructures or where it is not economically viable to offer terrestrial cellular coverage due to low population density.

The satellite component is a complementary part of the future cellular global transmission network, providing much larger service area but with smaller capacity than a terrestrial system. The design of the Satellite-Universal Mobile Telecommunication Systems (S-UMTS) architecture includes the choice of a satellite constellation, which determines the quality of service in terms of coverage area.

Although it may appear that satellite constellation achieves global coverage, but in reality path blockage represents a very critical problem especially at low elevation angles in particular for low earth orbit (LEO). To face this problem, satellite diversity with concatenated code can be introduce.

Diversity techniques coupled with DS-CDMA have proven very effective for terrestrial cellular systems, where the line-of-sight (LOS) signal is usually absent and Lognormal-Rayleigh fading is experienced. Furthermore, terrestrial system capacity is interference limited. In contrast, satellite systems are typically power limited and consequently forced to exploit the LOS signal. Additionally, the peculiar satellite system geometry and fading channel characteristics does not give any precious prediction of diversity benefits based on the results of the terrestrial cellular environments.

The objective of this study is exactly that of assessing the possible benefits achievable by exploiting satellite diversity. We focus on the impact of dual satellite diversity on system capacity, quality of services and service availability, by considering the down link system based on CDMA system with transparent transponders.

The adoption of CDMA with full frequency reuse and RAKE receiver is a very suitable way of exploiting satellite diversity, because it lowers the probability of dropping a call.

This paper deals with the bit error rate of CDMA-based multispot beam MEO \& LEO system with satellite diversity and imperfect power control for down link. In the analysis, the PCE is considered to be Lognormal distributed and the channe! is assumed to be Rician-Lognormal model.

The Paper is organised as follows. Limitation and requirements for designing NGEO satellite constellations are given in section two and the mobile satellite channel modelling is described in section three. Effect of imperfect power control is explained in section four and satellite diversity with Rake receiver in section five. System model is given in section six; simulation results and discussion are 
presented in section seven. Finally, some concluding remarks are given in section eight.

1- Limitation and requirements for designing NGEO satellite constellation

\section{1-Overview of LEO and MEO characteristics}

The renewed interest in the use of alternative orbits has been generated by the congestion in the GEO, propagation delay time and the problems associated with the use of very small terminals required in mobile applications. The interference to adjacent GEO satellites due to the wide-beam antennas normally used in the mobile terminals is unacceptable to the crowded GEO but does not present such serious problems to alternative orbits. Constellations of LEO and MEO satellite communication systems are used to provide giobal mobile satellite cornmunications. MEO mobile satellite communication systems have therefore been proposed, particularly to provide high elevation angle communication access at the northern latitudes. MEO systems typically are located at altitudes in the range from 10,000 to $16,000 \mathrm{~km}$ and with 6 hours periods. Typically $10-16$ satellites are used for global coverage and each satellite can only serve an area from 24 to $28 \%$

$$
10^{\circ}[3] \text {. }
$$

MEO systems increase the elevation angle of mobile satellite communications at. high latitudes (e.g. Europe) but still suffer from the high path loss and long time delays experienced in the GEO satellite systems. In order to overcome these problems, constellations of many LEO communication satellites have been proposed to provide global mobile communications to small terminals. The orbits of LEO satellites fall within the range $500-2000 \mathrm{~km}$ bounded by outer atmospheric drag and the start of the Van Allen radiation belts respectively. LEO systems require 20 to 100 satellites for worldwide services from elevation angles greater than $10^{\circ}$; each LEO satellite can only serve 2.5 to $5 \%$ of the Earth surface $[4,5]$. LEO satellite constellation communications systems appear well suited to global mobile applications. There is better satellite visibility (i.e., higher elevation angle), provision of satellite diversity (combats shadowing), lower altitude means shorter path (i.e., lower user terminal and satellite power), lower round-trip-delay (particularly important for mobile to mobile call), relatively low construction and launch cost per satellite, world-wide coverage and wide range of orbits.

Although there are new difficulties, such as varying link characteristics, handover from satellite to satellite, Doppler shift and fading in the use of LEC satellite communications, those are largely compensated by the reduction in the free space path loss, as well as the use of a multibeam antenna which makes it possible to use hand-held terminals in satellite mobile communications. In addition, the wide range of orbits avoids the congestion and interference experienced in the GEO. The lower satellite unit cost and graceful failure degradation of the LEO network are also attractive [6].

\section{2- Design parameters of satellite constellation}

The altitude of the satellite cannot be freely chosen, since the existence of two Van Allen radiation belts limits orbit selection. It is necessary to put the satellites out of these belts for minimizing the radiation damage to electronic components of the satellite. 
In designing LEO and other satellite constellations, there is a group of parameters to determine the performance and cost of that system. As the number of satellites used in the system is increased, the coverage area will also be expanded. But, unfortunately having a large number of satellites in the system means that costs of the operation and manufacturing will be large, in which turn will affect the cost of the services. This is also true in the case

service area. As for altitude, the higher altitude means the delay will be longer, which is important in the case of real-time communications. In design of a LEO system, the choice of satellite altitude is an important consideration, which has a significant effect on system performance. Among the factors affected by the satellite altitude choice are system capacity, user-to-user delay and power system design [7]. It is found that a choice of low altitude has a positive impact on system capacity and power system requirements. In terms of user-to user delay, the results are in favour of MEO. The overall propagation delay for telephone conversation is due to four main components:

- The propagation delay due to the distance from the satellite to earth (5-10 $\mathrm{ms}$ for LEO, 70-80 ms for MEO)

- The delay due to terrestrial networks (5-20 ms over land).

- The processing delay due to transmission systems.

- The processing time for voice coding and decoding (60-80 ms for a $4.8 \mathrm{kbit} / \mathrm{s}$ system).

The last component is independent of the type of satellite constellation and transmission system.

The design parameters of satellite constellation that need to be considered are as follows $[8,9,10]$ : number of satellites, number of orbital planes, number of satellite in each orbital planes, orbital height of satellite, inclination of the orbital planes, relative spacing of the orbital planes, relative phasing of the satellite in the same orbital plane, relative phasing of satellite in of adjacent orbital planes.

Elevation angle is very important as this parameter can be used, with the satellite altitude, to calculate a coverage area and visibility time of each satellite and therefore the whole constellation. For example, to change an elevation angle from $5^{\circ}$ to $10^{\circ}$, for satellite at altitude of $1,000 \mathrm{~km}$, the coverage area will change from $2.5 \times 10^{7}$ to $1.8 \times 10^{7}$ square $\mathrm{km}$ and the visibility time from 14.9 minutes to 12.64 minutes.

The choice of satellite altitude in a LEO system has a significant impact on the cost and performance of the system, and also has an impact on the expected satellite lifetime. Together, the number of satellites and their expected lifetime affect the system maintenance cost. The satellite altitude determines its maximum coverage area. The coverage area has two effects of interest in communication. The first effect is the maximum number of customers that can be serviced by each satellite. The second effect relates to frequency reuse and antenna technology. There is also a relationship between the satellite altitude and quality of service. Two generally accepted measures of quality of service are user-to-user delay and bit error rate. The bit error rate depends, among other factors, on the signal to noise ratio. 


\section{2- Mobile satellite channel modelling}

The Propagation channel plays an important role in the overall design of a mobile communication system. Shadowing of the line of sight (LOS) signal and multipath reception are the most significant issues of concern to system designers. Satellite mobile link quality is determined by the propagation channel characteristics. The propagation channel characteristics most significantly depend or the immediate environment surrounding a mobile. Signal amplitude variations of the order of several dBs may be experienced mainly due to the obstruction of the LOS path between a satellite and a mobile over a relatively short duration of time. There are several channel models that have been derived, describing the transmission path between a mobile/personal user and a GEO or NGEO satellite [11-13].

Due to the movement of the NGEO satellites, the geometrical pattern of shadowed areas is changing with the time. Similarly, the movement of the mobile/personal user translates the geometrical pattern of shadowed areas into a time series of good and bad channel states. The mean duration in the good and bad state, respectively, depends on the elevation angle, the type of the environment and the mobile user speed.

In hostile propagation environments, the channel will be subject to deep fades at some instant in time. The transmitted signal components corresponding to the propagation paths arrive at the receiver at different delay times.

Selection of accurate channel model represents a major challenge for this application, due to the large variety of environments in which the system must operate, and due to the fact that the link elevation angle changes continuously with the time. To account for both multipath fading and shadowing effects, the RiceLognormal channel model presented in [12] has been selected.

Although the channel characteristic varies over large areas, propagation experiments have shown that the channel characteristic remains constant over areas with identical environment features.

\section{3- Imperfect power control}

As far as the accuracy of the CLPC is concerned, the long round-trip delays (RTDs) associated with the satellite environments are the most limiting factor. This significantly limits the power control command Rate (PCCR) that in turn reduces the capability of the CLPC, in tracking the fast fading. However, the CLPC could still working well under circumstances such as a slow moving or a stationary UT.

It is more appropriate to consider the average elevation angles for the constellation for estimation of the average RTD, which changes with latitude. Table 1 shows the minimum and maximum round trip delay at minimum elevation angles for NGEO constellations systems [14].

At higher velocities interleaving becomes more efficient techniques in randomising the errors and hence maintain the required quality of services. On the other hand, increasing interleaving depths would introduce longer delays, which may not particularly suit a satellite system with an existing RTD. Therefore, striking the balance between the performance of the CLPC and interleaving for a given constellation becomes very important. For the standard IS-95 the transmitted power control bits are not encoded and hence do not experience any additional decoding delay. 
Table 1. Max. and Min. round trip delay at minimum elevation

\begin{tabular}{|l|l|l|l|l|}
\multicolumn{7}{c|}{ angle for NGEO constellation systems [14] } \\
\begin{tabular}{|l|l|l|l|l|} 
Altitude \\
$(\mathrm{km})$
\end{tabular} & Constellation & $\begin{array}{l}\text { Min.Elv. } \\
\text { Angle(deg.) }\end{array}$ & $\begin{array}{c}\text { Min.RTD } \\
\text { (ms) }\end{array}$ & $\begin{array}{l}\text { Max.RTD } \\
\text { (ms) }\end{array}$ \\
\hline 780 & LEO & 8.2 & 10 & 34 \\
\hline 1410 & LEO & 10 & 19 & 48 \\
\hline 10350 & MEO & 10 & 138 & 193 \\
\hline
\end{tabular}

In the uplink of DS-CDMA system, the problem of power control error represents the disadvantage point. The power control problem arises because of the multiple access interference. All users in the DS-CDMA system transmit the signal by using the same bandwidth at the same time and therefore users interfere with each other. Due to the propagation mechanism, the signal received by the satellite from a user terminal close to the satellite will be stronger than the signal received from another terminal located at the cell boundary. Hence the ciose user will dominate the distant users. To improve capacity, all signals, irrespective of distance, should arrive at the satellite station with the same mean power.

Power control attempts to achieve a constant received mean power for each user. Therefore, the performance of the transmitter power control (TPC) is one of several dependent factors when deciding the capacity of a DS-CDMA system [15].

In contrast to the uplink, in the downlink all signals propagate through the same channel and thus are received by a mobile station with equal power. Therefore, no power control is required to eliminate the near-far problem. The power control is, however, required to minimize the interference to the other cells and to compensate against the interference from other cells. The worst-case situation for a mobile station occurs when the mobile station is at the cell edge. However, the interference from other cells does not vary very abruptly. Furthermore, power control improves the performance of DS-CDMA against fading channels by compensating the fading dips. If it followed the channel fading perfectly, power control would turn a fading channel into channel close to AWGN channel by eliminating most of the fade dips.

The effect of the power control imperfections is investigated considering the results reported in [16], which refer to different values of standard deviation of PCE, ranging from $0-3 \mathrm{~dB}$.

By inspection of the results, we can confirm the well-known sensitivity of DS-CDMA to power control accuracy. As a consequence, a thorough evaluation of system performance cannot be carried out neglected the effects of power control imperfections.

\section{4- Satellite Diversity with RAKE Receiver}

A spread spectrum signal waveform is well matched to the multipath channel. In a multipath channel, the original transmitted signal reflects from obstacles such as buildings and mountains, and the receiver receives several copies of the signal with different delays. If the signals arrive more than one chip apart from each other, the receiver can resolve them. Actually, from each multipath signal's point of view, other multipath signals can be regarded as interference and they are suppressed by the processing gain. 
In terrestrial cellular communication systems, the mobile user rarely has LOS to the base station. This implies that the mobile terminal would rely on the multipath reflection and echo signal most of the time for communication. In relatively narrowband communication systems, the presence of the multipath signal causes severe fading and deterioration of the quality of services. In contrast, in wideband CDMA systems, the multipath signal can be exploited efficiently by using RAKE receiver, which allows the resolved signals arriving with different propagation delays to combine and provide an additional gain. The use of RAKE receivers in terrestrial system proves to be very efficient due to the sufficient delay spread associated with the channel.

However, in the satellite mobile environment this is not as straightforward due to small delay spreads associated with such environments. In satellite channels the delay spread has an average about 100 nsec. Hence any CDMA system designed to combat the multipath would have to be spreading by at least an arnount greater than the coherence bandwidth (i.e., $10 \mathrm{MHz}$ or more). Even if the multipath is resolved, the echoes arrive at much lower power compared to the LOS signal that limits any multipath diversity gain. Nevertheless, RAKE receivers are still useful for combining the received signals from visible different satellite diversity at the same time; the link margin can be reduced by approximately $6 \mathrm{~dB}$ with respect to single satellite visibility especially at low elevation angles [17].

Figure (1) shows an example for Globalstar satellite visibility of a LEO constellation system. As can be seen in the figure, two or more satellites are visible between $25^{\circ}$ and $50^{\circ}$ latitude at $100 \%$ of the time, and from the equator to $60^{\circ}$ latitude $80 \%$ of the time.

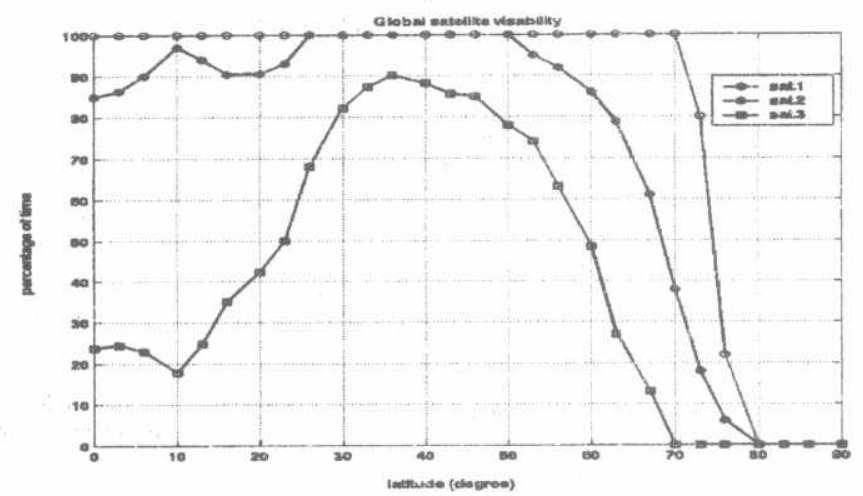

Fig.1. Global satellite visibility of LEO constellation systern

5.1-The effect of satellite diversity on service availability

The availability and quality of services in satellite systems is crucially influenced by the particular characteristics of signal propagation in the link between the mobile or personal user and the satellite. In a particular NGEO satellite scenario, the behaviour of the channel and the parameters of any shadowing and fading process 
for a channel model are expected to be closely coupled with the varying elevation angle of the mobile user link.

In this crucial shadowing and fading environment, satellite diversity can act as an efficient countermeasure against QOS degradation by exploiting multiple satellite visibility and providing simultaneous communication via two or more visible satellite. System availability of mobile satellite communications depends strongly on an unobstructed line of site to one or more of the mobile satellite in the coverage area of the mobile user.

The major concern arises from fading at low elevation angles, and operation at low elevation angles is unavoidable sometimes with LEO systems. The user terminal antenna will have essentially omni direction coverage, and will be subject to terrain and other obstacle blockage at low elevation angles. The provision of an unobstructed line of sight between mobile user terminal and a satellite depends in turn on the possibility of high elevation angles.

From [18-19], the results appeared to indicate that in order to achieve an availability comparable to that of well-developed cellular system, at least $95 \%$, it would be necessary for single satellite systems to provide substantial margin at low elevation angles, or to modify the satellite constellations so that elevation angles above $40^{\circ}$. For operation down to about $10^{\circ}$ elevation angle, it appears that a margin of 15 to $20 \mathrm{~dB}$ would be provided to achieve 95 to $98 \%$ availability. For an $30^{\circ}$ elevation angle the required margin is still about $12 \mathrm{~dB}$, while only $40^{\circ}$ does the requirement fall below $10 \mathrm{~dB}$.

However, another approach adopted by utilizing path diversity and this is possible for constellation with a large number of satellites and suitably chosen orbital parameters.

Then if the path to one satellite is obstructed the probability of an unobstructed path to another satellite is high. Using this approach the required power margin is reduced to $5-7 \mathrm{~dB}$.

For example, the Odyssey system (MEO) has elevation angles as high as $50^{\circ}$ during the course of transmission. Because a satellite is in view for about two hours in the Odyssey system, the angle to satellite will change slowly during the duration of a call, reducing the chance of the satellite moving behind an obstruction. The forward link budget shows an average downlink margin of $6.3 \mathrm{~dB}$ that includes propagation loss and self-interference from the CDMA modulation format.

Also, Globalstar plans to tackle this problem by the use of path diversity, making use of transmissions through two or three satellites both in the forward and return directions. The predicted amount of fade margin required for a receiver for shadowed propagation through trees varies with latitude, typically ranging from less than $5 \mathrm{~dB}$ up to $7.5 \mathrm{~dB}$ for $98 \%$ percent availability

Figure (2) shows an example of availability of LEO satellite constellation at height $1400 \mathrm{~km}$ and covered the population area until $\pm 70^{\circ}$ latitude for urban environment. Due to the high speed of satellite in LEO (i.e.; $7 \mathrm{~km} / \mathrm{s}$ ), it is better to use satellite diversity to alleviate performance impairment due to blockage. Figure (3) shows average elevation angle of two satellites with different latitude for LEO constellation. 


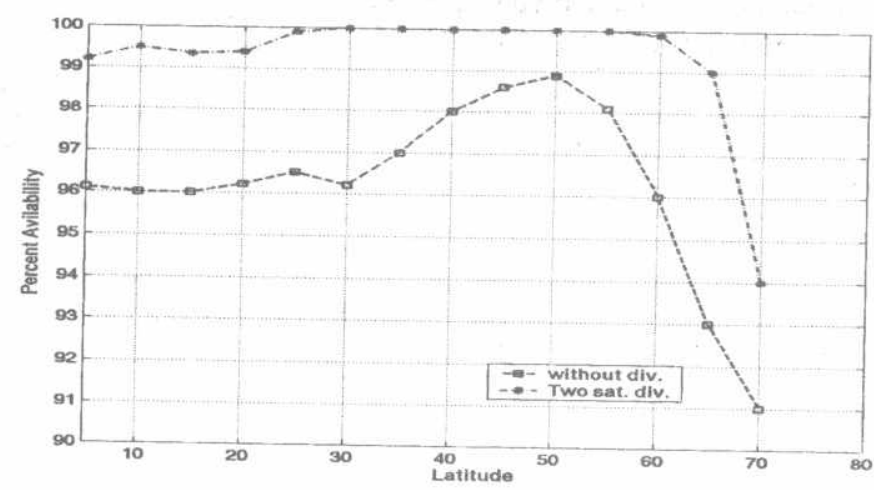

Fig.2. Availability in rural environment with satellite diversity

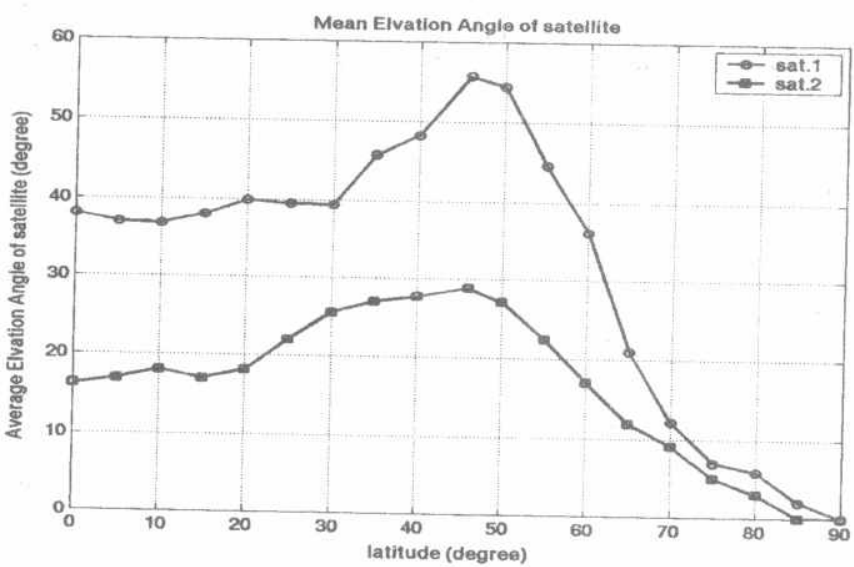

Fig.3. Mean Elevation angle of satellite with different latitude

We conclude that the availability issue has been recognized as one of the primary system design issues by each proposer. The techniques proposed to alleviate performance impairment due to blockage include multiple satellite signal combining, high elevation angles, increased power margin and combinations of these.

\section{2- Probability of Call dropping}

The service area of the spotbeam varies with the time, according to dynamic movement of the satellite in the constellation. Hence the service link is transferred from spotbeam to another spotbeam. When the mobile is out of satellite coverage area or another satellite with higher elevation angle is available, the link is transferred to the next satellite.

The maximum quality of service, expressed in terms the probability of call dropping, can be achieved by exploiting the available satellites, using RAKE receiver. 
The availability of multiple satellites is an essential condition to decrease probability of call dropping but it is not sufficient. We need also to have a soft handover from spotbeam to another and from satellite to another satellite.

In this case the CDMA is the unique option for providing soft handover (i.e., full frequency reuse) capability with RAKE receiver to reduce the probability of call dropping. This is very significant in terms of reducing the probability of call dropping relative to hard handover from one frequency channel to another. Furthermore no frequency management is required and it simplifies the RF interfacing.

\section{3- Blockage probability}

Under the assumption that the blockage of signal from different satellite links is independent, we define blockage probability from a number of visible satellites as follows;

$P_{b}=\frac{1}{(N F)^{N_{s}}} \prod_{m=1}^{N_{s}}\left(90-\alpha_{m}\right)^{2}$

Where $\alpha_{m}$ is the elevation angle for the $\mathrm{k}$-th satellite in view from the range $\left(10^{\circ}\right.$ to $\left.90^{\circ}\right)$, and NF is the normalization factor, which is changed according to the environments ( $\mathrm{NF}=7000$ in urban area and 16000 in suburban area) [20].

From figure (4) in the left side, the path blockage probability for the urban area is very high. For examples, by setting elevation angle $\left(\alpha_{m}\right)=30^{\circ}$, blockage probability is approximately $52 \%$ with $\mathrm{Ns}=1$, but with dual satellite diversity the blockage probability reduces to $27 \%$ and for case of $N s=3$ the blockage probability reduces to $14 \%$. In the right side of figure (4), the situation is different for path blockage probability for suburban area. For example, at $\alpha_{m}=20^{\circ}$, blockage probability is typically $32 \%$ with $\mathrm{Ns}=1$, but with dual satellite diversity the blockage probability reduces to $9 \%$ and for case of $\mathrm{Ns}=3$ the blockage probability reduces to $3.5 \%$.
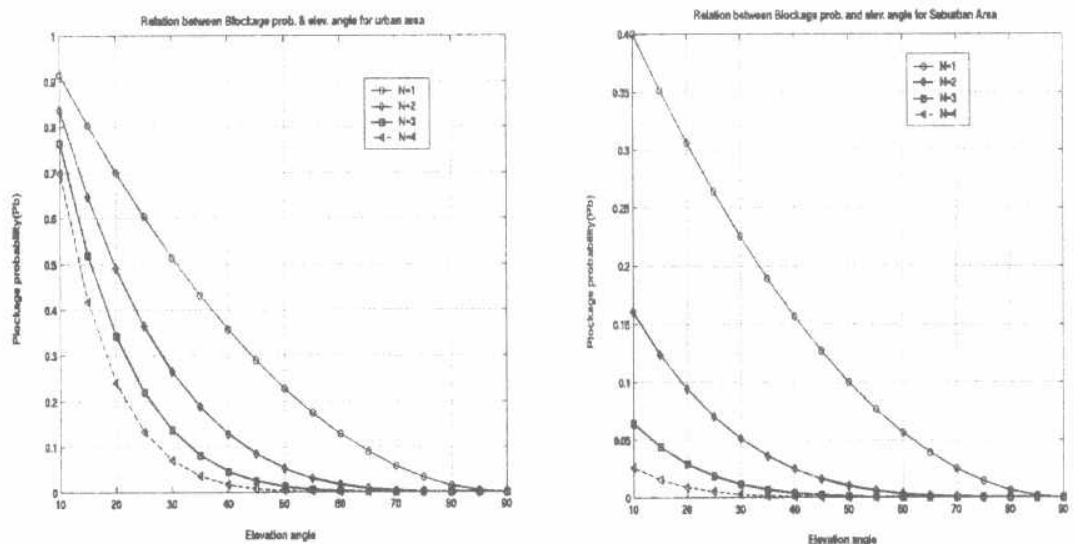

Fig.4. Blockage probability and elevation angle for Urban and Suburban areas 
The summary from this discussion shows that satellite diversity is a necessary condition in providing service availability particularly for suburban and urban areas. This means that the designer must consider satellite diversity especially in populated latitudes.

\section{5- System model}

The system is based on a NGEO satellite constellation providing global coverage and large probability of multiple satellite visibility. The satellite is equipped with multibeam antenna and transparent payloads. The analysis is applied for the case

coder and Reed Solomon $(255,243)$ code as an outer code and Rake receiver with maximal ratio combining.

We derive the capacity of the system under different elevation angles and we average the capacity under percentage of time the satellite in view at this elevation angle. Due to the long round trip delay, the power control system cannot track the variation in the channel perfectly and for this reason we assume the effect of power control error equals $1 \mathrm{~dB}$. Furthermore, we consider the effect of voice activity factor, which reduces the MAI.

The Rice-Lognormal channel with different Rician factor and standard deviation error are applied according to the different elevation angles. We assume that all sequences within a given spotbeam are orthogonal and processing gain is $P G=64$, defined as the number of chips per coded symbol and perfect interleaving is considered. The two satellites transmit the signal with different carriers to maintain signal independence.

\section{6- Simulation Results}

In this section, simulation results are shown of the performance of DS-CDMA systems, operating over a rural area with tree shadowing according to the Corazza model [12] with satellite diversity.

BER is plotted against the number of users per spotbeam per carrier at different elevation angles according to latitude of user terminal. If we assume the standard deviation of the power control error is $1 \mathrm{~dB}$, and we choose a BER of $10^{-3}$ as our goal at $E b / N o=6 \mathrm{~dB}$. In figure (5), for Low elevation angle $20^{\circ}$ and $30^{\circ}$ the performance of the system is poor. For example, the system can accommodate about $\mathrm{M}=2$ and 6 users per spotbeam per carrier respectively.

When the elevation angle starts to increase above $40^{\circ}$ the performance of the system improves very well. For example, in figure (5) the system can accommodate about $M=19,24,27,28,30$ and 31 users per spotbeam per carrier respectively for elevation angles $40,50,60,70,80$ and $90^{\circ}$ respectively. Due to high relative speed of the satellite at high elevation angles, the satellite stays a very short time at high elevation angles and stays $44 \%$ of the time at elevation angle between $40^{\circ}$ and $50^{\circ}$.

Table (2) shows a statistics for two different constellations discredited probability density function for Elevation angles at certain latitude. In table 2, there are two examples for MEO and LEO satellite Constellations. It is clear that LEO satellite 
constellation stays approximately $44 \%$ at elevation angle between $40^{\circ}$ to $50^{\circ}$ and $88.5 \%$ at elevation angle between $30^{\circ}$ and $60^{\circ}$. But MEO satellite corrstellation stays $90 \%$ at elevation angle between $30^{\circ}$ and $80^{\circ}$.

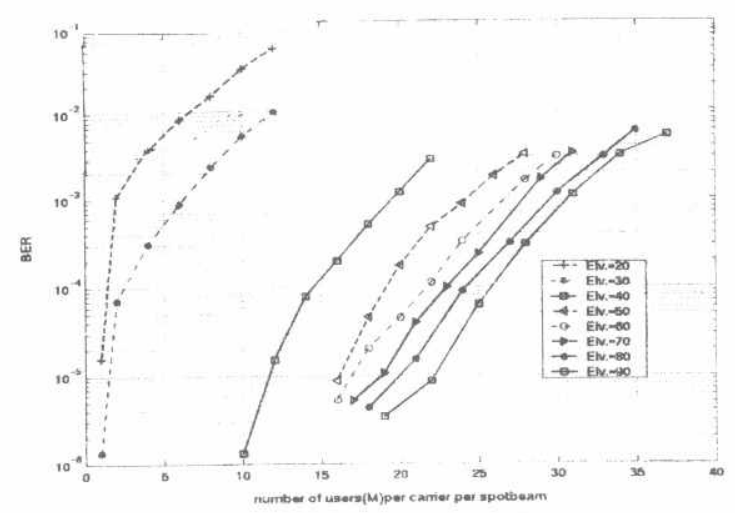

Fig.5. Relation between Number of users per spot beam per carrier and BER

Table 2. Discredited PDF for Elevation angles at (42 N Lat., $12 \mathrm{E}$ Long.) [12].

\begin{tabular}{|l|l|l|l|l|l|l|l|l|}
\hline Elv. (deg.) & $90-80$ & $80-70$ & $70-60$ & $60-50$ & $50-40$ & $40-30$ & $30-20$ & $20-10$ \\
\hline $\begin{array}{l}\text { Odyssey } \\
\text { (10350km) }\end{array}$ & 4.8 & 16.9 & 19.4 & 19.0 & 19.6 & 15.1 & 5.2 & 0.0 \\
\hline $\begin{array}{l}\text { Globalstar } \\
(1400 \mathrm{~km})\end{array}$ & 0.4 & 2.6 & 8.4 & 22.7 & 44.0 & 21.9 & 0.0 & 0.0 \\
\hline
\end{tabular}

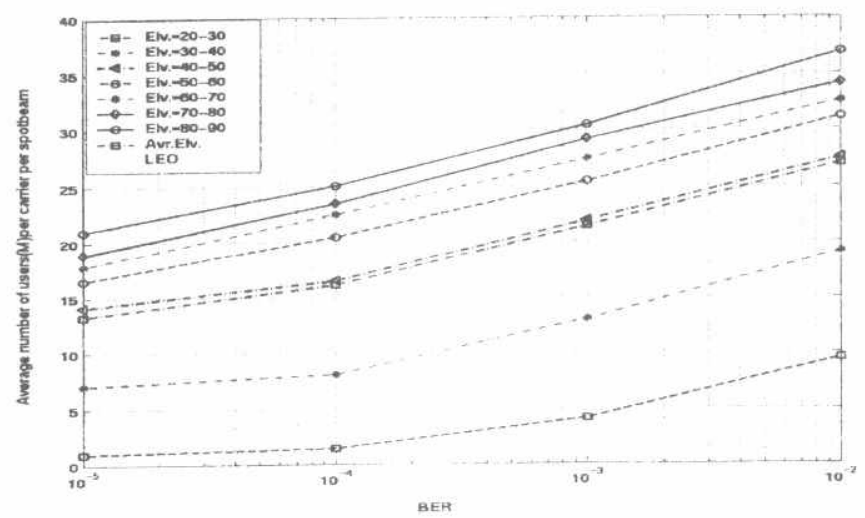

Fig.6. Capacity of LEO per spot beam per carrier 
Figure (6) draws the relation between the number of user per spot beam per carrier and BER for LEO satellite constellation system as an example. If we take the average for the number of users user per spot beam per carrier, the results show LEO can offer 21 users, this is close to the elevation angle between $40^{\circ}-50^{\circ}$, and this result agrees with the statistics of LEO in table 2.

Fig.7 draws the relation between the number of user per spot beam per carrier and $B E R$ for MEO satellite constellation system as an example. If we take the average for the number of users user per spot beam per carrier, the results show MEO can offer 23 users and this is greater than the number of users at elevation angle between $40^{\circ}-50^{\circ}$. Furthermore, this result agrees with the statistics of MEO in table 2.

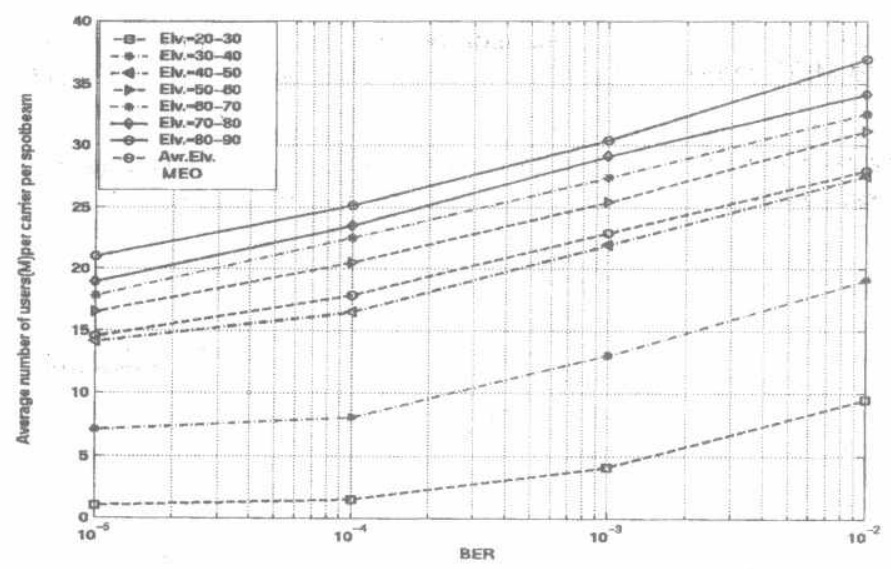

Fig.7. Capacity of MEO per spot beam per carrier

If we fix the number of user per spot beam per carrier at 22 users and measure the performance at $\mathrm{BER}=10^{-3}$, fig. 8 shows the required $\mathrm{Eb} / \mathrm{No}$ at different elevation angles. For example users at elevation angle $=90^{\circ}$ requires $4.8 \mathrm{~dB}$ to achieve the requirement, but the same number of users at elevation angle equals $20^{\circ}$ need $12.4 \mathrm{~dB}$ to achieve the same requirement (i.e.; $7.8 \mathrm{~dB}$ more than users at elevation angle $90^{\circ}$ ). For a real comparison with respect to average elevation angle of LEO $\left(40^{\circ}-50^{\circ}\right)$ as given in table 2 and fig. 6 , the users at $20^{\circ}$ elevation angle needs $6.4 \mathrm{~dB}$ more power to achieve the same performance.

This results shows that the user at low elevation angle needs more power to compensate path loss, shadowing and fading and the designer must consider the fade margin to maintain the performance of the system. This value may be double in the case of a single satellite. 


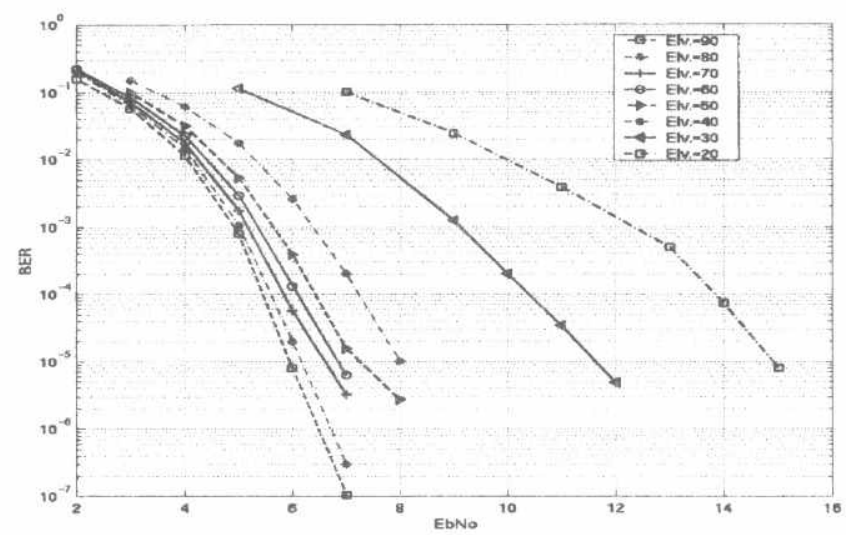

Fig.8. Performance of the system over different elevation angles

Figure (9) shows the comparison between the performance of an example of LEO and MEO satellite constellation. The LEO requires approximately $6.18 \mathrm{~dB}$ to achieve the performance of 22 users at $B E R=10^{-3}$, but MEO requires $5.9 \mathrm{~dB}$ to achieve the same requirement. If our objective is $B E R=10^{-5}$ for transmit data the $L E O$ in this example requires $7.7 \mathrm{~dB}$ to achieve requirement, but $\mathrm{MEO}$ requires $7.45 \mathrm{~dB}$ to achieve the same requirement.

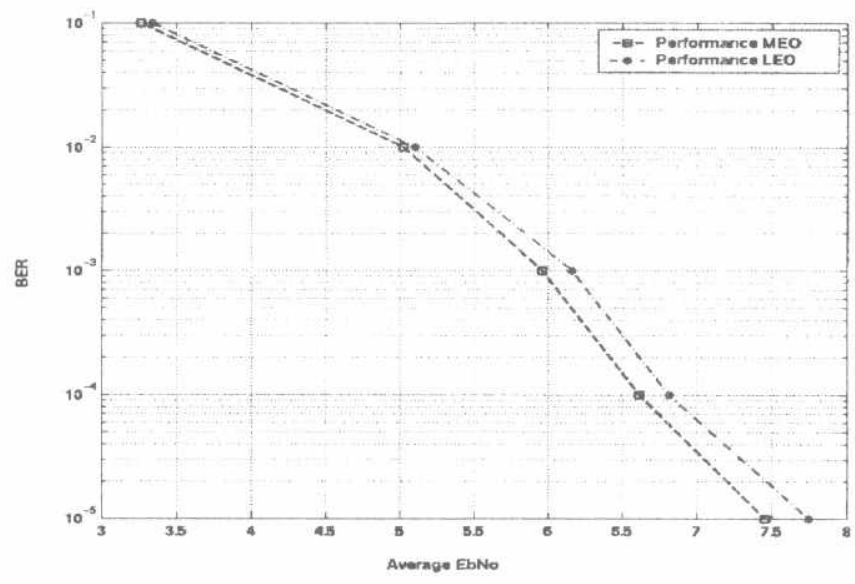

Fig.9. Performance of LEO and MEO satellite 


\section{7- Conclusion}

In this paper the impact of satellite diversity on both services availability and system capacity has been investigated by means of analysis and simulation model, taking into account the effects of fading channel characteristics, the effect of link blockage and power control imperfection. We can draw the conclusion that satellite diversity is an essential factor to achieve a satisfactory level of service availability and quality of service. A significant improvement in services availability was achieved by using highest two satellites.

Satellite diversity should always be advantageous in dimensioning the probability of call blockage and generally improve transmission quality and capacity of the system. The reduction of probability of call dropping can be achieved by exploiting the satellite diversity with full frequency reuse, based on CDMA.

The average capacity per carrier per spotbeam changes slightly with elevation angle below $30^{\circ}$, but the significant variation in the capacity is clear for elevation angles above $40^{\circ}$ and the capacity for dual satellite diversity varied with visibility statistics of the system.

The capacity of MEO per spot beam per carrier is greater than LEO, however the capacity of LEO satellite constellation is greater than the capacity of MEO satellite constellation due to the large number of satellites in LEO constellation compared to number of satellites In MEO constellation for global coverage.

The best gain in service availability can only be achieved if the considered satellite channels are independent. Therefore, any dependency between the satellite channels influences the advantage of the satellite diversity. The effective correlation of the two channels is highly dependent on the elevation angle and azimuth separation of the two satellites with respect to the user and the operational environment.

\section{8- Reference}

[1] R. L. Picholtz, D. I. Shilling, and L. B. Milstien, " Theory of spread spectrum communications-a tutorial," IEEE, Trans. communication, Vol. com-30, No.5, (1982).

[2] Jhong S. Lee, Leonard E. Miller, "CDMA systems engineering handbook," Artech House, (1998).

[3] R. Nelson, "Satellite constellation geometry," via satellite, pp.110-143, vol.5, No.3, (1995)

[4] M.Richharia and et al, " A feasibility study of mobile communications network using a constellation of LEO satellites," IEEE GLOBECOM'89, Dallas, 27-30
November (1989).

[5] A.Boticher, A. Jahn, "Analysis of Basic System Parameters of Communication Networks Based on Low Earth Orbit Satellites," International journal of satellite communications, vol. 12, pp. 85-93, (1994).

[6] Richard A. Raines, Nathaniel J. Davis, "Personal Communications via low Earth orbit satellite communication networks," IEEE 1995, pp. 1229-1233, (1995).

[7] B. Gavish, "Height Considerations in Low Earth Orbit Satellite Systems," Institute of industrial engineers $4^{\text {th }}$ industrial engineering research conference Proceedings, pp. 1047-1057, (1995). 
[8] Jose Radzik, G. Maral, "A Methodology for Rapidly Evaluating the Performance of Some Low Earth Orbit Satellite Systems," IEEE journal on selected areas in Communications, vol. 13, No. 2, Feb.1995, pp. 301-309, (1995).

[9] B. Gavish, "LEO satellite based communication systems research opportunities," European Journal of Operational Research99, pp.166-179, (1997).

[10] D.C. Beste, "Design of Satellite Constellations for Optimal Continuous overage," IEEE transactions in aerospace and electronic systems, aes-14, No. 3, May 1978, pp.466-473, (1978).

[11] M. Parks, B.G. Evans, "Simultaneous wide-band propagation measurements applicable to mobile satellite communication systems at $L$ and s-band," AIAA-961093-CP, pp.929-936, (1996).

[12] Giovanni E. Corazza, and F.Vatalaro, "A statistical model for land mobile Satellite channels and its application to non-geostatinory orbit systems," IEEE Transaction on vehicular technology, vol.43, No.3, PP 738-741, (1994).

[13] E.Lutz, Daniel Cyan, "The Land Mobile Satellite Communication Channelrecording, Statistics, and Channel Model," IEEE transactions on vehicular Technology, vol. 40, No. 2, pp. 375-385, (1991).

[14] P. Taaghol, S. Nourizadeh, R. Tafazoli, " An advanced power control scheme for CDMA-based satellite communication systems," Sixth International mobile satellite conference, Ottawa, June1999, pp.180-184, (1999).

[15] A.M.Monk, L.B.Milstein, " Open-loop power control error in a land mobile satellite system," IEEE Journal on selected areas in Communication, vol.13, pp.205-212, (1995).

[16] T.Attia, P. Sweeney and M.Sweeting, "Effective factors on the performance of LEOS, based CDMA systems," Proceeding of the $15^{\text {th }}$ AMSAT-UK Colloquium, University of Surrey, pp. $37-46$, July $28^{\text {th }}-30^{\text {th }},(2000)$.

[17] T.Attia, P.Sweeney and M.Sweeting, "Performance of DS-CDMA downlink with dual satellite diversity for mobile satellite communications," Proceedings of IST mobile communications Summit 2000, Galway, Ireland, 1-4 Oct. 2000, pp.65-71, (2000).

[18] L.M. Gaffney, " A re-evaluation of selected mobile satellite communications systems: Ellipso, Globalstar, Iridium and Odyssey", ESAESTEC sponsored report by MITRE, MTR930000157, Feb. (1994).

[19] E. Hirshfield, "The Globalstar system: breakthroughs in efficiency in microwave and signal processing technology, "Space communications14, pp.69-82, (1996).

[20] Y.Karasawa et al., "Analysis of availability improvement in LMSS by means of Satellite diversity based on Three-state propagation channel Model, ${ }^{\text {" IEEE Trans }}$ on Vehicular Technology, Nov. (1997) 